\title{
Development of Anxiety and Depression Inventory for Secondary School Students in Iran (A \& D Inventory)
}

\author{
Mahnaz Fallahi Khesht-Masjedi, ${ }^{, *}$ and Zoharah Omar ${ }^{2}$ \\ ${ }^{1}$ Psychology Department, Guilan University of Medical Scienses, Shafa Hospital Psychiatric Research Center, Rasht, IR Iran \\ ${ }^{2}$ Faculty of Educational Studies, University Putra Malaysia, Malaysia \\ "Corresponding author: Mahnaz Fallahi Khesht-Masjedi, Guilan University of Medical Scienses, Shafa Hospital Psychiatric Research Center, 15 Khordad Avenue, Rasht, IR Iran. \\ Tel: +98-9118392125, +98-1333334480, E-mail: mahnaz.fallahi@gmail.com
}

Received 2015 July 28; Revised 2015 December 31; Accepted 2016 September 23.

\begin{abstract}
Background: Currently clinicians and researchers in Iran have to use translated western instruments to screen for anxiety and depressive disorders. Although, some western questionnaires translated and adapted for Iranian people have demonstrated suitable validity in Iranian context, considering the difference in the cultural values, it still remains unclear as to whether a realistic picture of this phenomenon in Iranian society has been drawn. Hence, for detecting and monitoring common emotional disorders in Iran, researchers need to reflect the way that young people experience and express this disorder in the Iranian culture.

Objectives: This study seeked to address these shortcomings by developing and validating an instrument to measure anxiety and depression among adolescents in Iran with the Tripartiate Model and cognitive content specificity theory serving as the conceptual basis for the instrument development.

Materials and Methods: The participants constituted of 579 students from secondary schools, who resided in the north of Iran during years 2011 and 2012. The instrument was developed and validated using exploratory factor analysis (EFA) (Phase II) and confirmatory factor analysis (CFA) (Phase III) to ensure its reliability and validity.

Results: The results provide evidence that the developed instrument achieved sound psychometric properties. The hypothesized two-factor model identified via EFA and CFA in the study consisted of 32 items, with 16 items belonging to anxiety and 16 items to depression. The overall reliability value of Cronbach's Alpha was 0.87 . The CFA results showed acceptable goodness-of-fit indices for the revised model.

Conclusions: The anxiety and depression (A\&D) inventory is a simple, brief, and easy to administer tool. Indeed, the entire 32-item $A \& D$ inventory takes no longer to complete than other symptom measures. Therefore, the A\&D Inventory can provide differentiated symptoms between anxiety and depression in a very quick and efficient manner for Iranian adolescents.
\end{abstract}

Keywords: Anxiety, Depression, Development, Student, Validation

\section{Background}

The adolescence years are a time of transition from childhood to adulthood. These years are important as an adolescent asserts his or her individuality. Many adults may encounter adolescents, who seem constantly unhappy, show little enthusiasm for anything, are moody, or, at worst, think life is not worth living, and these are often regarded as mild and transitory disturbances experienced by almost all adolescents, which would fade away as they move into adulthood (Marsh and Wolfe). In reality, these adolescents may have emotional disorder (1). Often when an adolescent has been diagnosed with emotional disorder, they are diagnosed to have anxiety and depression (2). One way that helps recognize emotional disorder is through psychological assessments. Since most adolescents will not talk about their problems very easily, information and data obtained from psychological assessment may provide a scientifically more reliable assessment than the information from a clinical interview (3).

Researchers in Asian countries including Iran have to use translated western instruments to screen for anxiety and depressive disorders. Although, some western questionnaires translated and adapted for Iranian people have demonstrated suitable validity in the Iranian context, considering the difference in cultural values of Western cultures and the Iranian culture it still remains unclear as to whether a realistic picture of this phenomenon in Iranian society has been drawn (4) when using the Western instruments for detecting and monitoring common emotional disorders in Iran. For development of any new questionnaire, careful attention needs to be given to the language and idiom of emotional distress in Iran (5). Cultural differences in societal structures, values and socialization practices may contribute to the differences found in the meaning and/or structure of a measured construct and the perception of its related item content. Thus, societal quali- 
ties alone can contribute to structural and measurement nonequivalence $(6,7)$.

Mental health in Iran is an essential health issue. It needs to be systematically and continuously studied (8). It was reported that in Iran the rate of emotional disorders was $19.5 \%$ and the prevalence of emotional disorders tend to increase among girls up to $34.1 \%$ higher than boys at $27.7 \%$ as they move through childhood and adolescence $(4,9)$. Based on some studies on independent samples in Iran, a significant number of adolescents are at risk of various emotional problems $(10,11)$ and at risk of attempting self-harm and suicide (12). The essential tensions experienced by youth should be studied and solutions to prevent them should be proposed to the authorities and be implemented (13).

However, cross-cultural studies on young people's anxiety and depression symptoms have been relatively ignored $(14,15)$. Self-reporting of anxiety and depression has been the basis of clinical research for more than 40 years. Researchers can choose from several self-report scales, but at this time, the accumulating research has exposed some limitations in these instruments, and identified the need to develop alternative measures (16-19).

Ironically, after an online search of databases, it was discovered that, a few past researches have examined factors such as anxiety and depression among Iranian adolescent. One reason is that, there is no suitable valid instrument to measure emotional disorders like anxiety and depression for adolescents. Therefore, this study sought to address these shortcomings by developing and validating an instrument to measure anxiety and depression among adolescent in Iran using two theories: first, the Tripartite Model, and second cognitive content specificity (Figure 1).

\section{Materials and Methods}

Design: This research design adopted a quantitative approach in order to accomplish this purpose. A multiphase approach was used, which included item generation (Phase 1), item analysis (Phase II) and finally crossvalidation (Phase III).

\subsection{Phase I}

Items generation: item pool generation was guided by the conceptualization of anxiety and depression in a tripartite model and the cognitive content specificity (CCS) theory $(20,21)$. The Tripartite Model explains both the overlapping and distinct features of anxiety and depression and has been used to describe the relationship between distress disorders and personality dimensions (22). In the CCS theory, depressive cognitive content is related to negative self-evaluation, hopelessness and generally pessimistic assessments of the world. Meanwhile, the anxious cognitive content was proposed to be related to thoughts concerning physical or psychological threats (23). The instrument development during this phase involves five stages as described below:

1) Item writing: at this stage several issues were considered such as: wording clarity, wording redundancy, positively and negatively worded items and choice of response format (24). In this early stage of the instrument, called the "A\&D Inventory-p" (A\&D Inventory pilot), large item pools for the constructs of anxiety and depression were generated from the literatures. The A\&D inventory-p was arranged in a format designed to elicit a rapid flow of item responses. The purpose of this new format design was to increase the rate of participant compliance in phase 2 and to strengthen the administrative utility of the final version of the "A\&D Inventory". The items were worded in Persian language used by adolescents about their anxiety and depression, and incorporated into a self-reporting measure with a five-point response scale, ranging from "strongly disagree" to "strongly agree". A total of 158 items were generated in which 67 items measured anxiety and 91 items measured depression.

2) Item response format: although there was no agreement as to which item format is most optimal (21), choosing a suitable item response format is important (24) because it can influence the variability of the scale response. The use of ordinal scales with too few categories (less than five) can result in distorted findings (25). Aside from expanding the number of items in the scale, adopting a response format that has higher number of scale response categories may increase variability (26). A five-point response scale, ranging from (1) "strongly disagree" to (5) "strongly agree", was chosen as the item response format in this study.

3) Items selection: this aimed at enhancing the content validity of the scale. Content validity depends on the extent to which a measure reflects a specific domain of content (27), as well as on the adequacy with which this domain has been sampled and cast in the form of test items (28). There are no agreements upon the criteria for determining the extent to which a measure has attained such validity. Usually, a general accepted procedure for testing content validity is to obtain a consensus from expert judges about the extent to which the instrument reflects the domains defined by the theory, and the degree to which this content is adequately sampled and cast in the form of test items (24).

4) Initial item testing and refinement: At this point, review of the item pool had just begun. The steps to follow included content validation and further qualitative evaluation in which the quality of the items was assessed in re- 


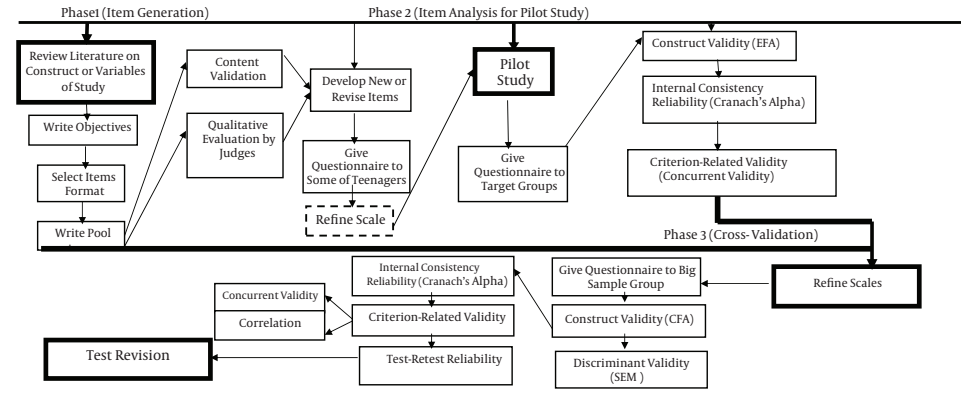

Figure 1. Process of Instrument Development

lation to the target group. Items that did not attain the required standard even after several revisions were dropped. At this stage the researchers' focus was not so much on the actual responses but the items and the procedures for answering the questionnaire, which were then discussed item by item. By doing this, the researcher was able to understand how the respondents reacted to the items and what meanings they gave so that they could be compared to the original meanings the researcher had in mind when the items were generated.

5) Selection of the final item: altogether, a total of 158 items were chosen with 67 items that measured anxiety and 91 items that were related to depression. To ensure content validity, all questions related to anxiety and depression were sent to professional psychologists and psychiatrists (a PhD in clinical psychology, a professor of psychiatry, a MD of child and adolescents psychiatry and a PhD in counseling) in Iran for content validation. In addition, we selected 60 adolescents to read the items, encouraging them to ask for explanations should they not understand any of the items. This particular step usually leads to various adjustments and changes arising from new insights gleaned from the discussion (29).

\subsection{Phase $\Pi$}

Item Analysis in Pilot Study: A good research strategy requires careful planning and a pilot study will often be a part of this strategy (30-32). The goal of Phase 2 of this study was to produce a shortened psychometrically refined instrument for measuring anxiety and depression by employing item analysis procedures on collected A\&D Inventory-p response data. The nature and quality of the items in the initial pool were also assessed during this phase. For example, the researcher was concerned whether the items in the A \& D Inventory-p related too strongly or too mildly to the variable. The following procedures were executed during this phase.
1) Reliability analysis to test the internal consistency of the items using:

a. Corrected item-total correlation: From this test, if the score of 'corrected item total correlation' is less than 0.5 , the item should be removed or reworded. On the other hand, if the score exceeds 0.3 , the item is proven to be working properly (33).

b. Alpha if item deleted: Based on the test, if an item's 'alpha if item deleted' value is greater than the standardized item alpha score, the item should be deleted immediately (33). At this stage, 300 adolescents were selected for the pilot study (as explained in the sample size of the pilot study). In this phase, the samples completed filling in the new instrument (A\&D inventory-p) with two of the selfreporting scales validity and reliability, in Iran (BAI, BDI) for criterion validity.

2) Validity: The aim at this stage was to test the construct validity of the A\&D inventory-p using exploratory factor analysis. The criterion validity of the A \& D inventory-p was also tested to determine how well the A\&D Inventory-p correlates with a previously validated measure.

3) Revision: based on item analysis, the test was refined, retried, analyzed and refined. During revision, some items were eliminated.

\subsection{Phase III}

Cross-validity: This phase was implemented with a bigger sample size so that various statistical analyses, including confirmatory factor analysis (CFA) using structural equation modeling (SEM), were carried out. In this phase, the samples filled the new instrument with two of the selfreporting scales of validity and reliability in Iran (BAI, BDI). The researcher then collected and analyzed the collected data.

\subsection{Samples}

The population of this study comprised of adolescents in the north of Iran. From the population, two different 
samples were drawn for the Pilot Study phase (Phase II) and cross-validation phase (Phase III), respectively.

Sample in Phase II: a total of 300 adolescents were selected during this phase. For this stage, distribution of samples was selected from five cities in Gilan province. At first, the list of Guilan health centers was obtained from the vice-chancellor for health of Iran Guilan University of Medical Sciences (GUMS). The number of households covered by each health center was calculated and clusters were randomly chosen from households of five urban and five rural areas. Male and female subjects were not selected separately. Selecting the location of the first cluster was based on a random number chart. Next, using a systematic sampling technique knowing the inter cluster interval, subsequent clusters were selected. The researcher and her colleagues met the study samples and explained the aim of this research. Individuals with any communication problem, mental retardation, chronic schizophrenia, and those who did not respond for any reason were excluded.

Sample in Phase III: Cluster sampling was also used at this stage, as it is a sampling technique in which the whole population of interest is divided to groups, or clusters, and a random sample of these clusters is selected (34). During this phase, a total of 579 schools with 308 males and 271 females from 16 cities of Guilan province were selected. Their ages ranged from 13 to 19 years and were from different grades. At the beginning of the test, the researcher explained the assessment to the samples and they were made aware of the objectives of the research in this study by the researcher.

\subsection{Instruments}

Two instruments were used in this study to test the validity of the new instruments (A\&D inventory): 1-Beck anxiety inventory (BAI), and 2- Beck depression inventory (BDI).

Beck anxiety inventory (BAI): the Beck anxiety inventory (BAI), developed by Beck and colleagues, is a 21-item multiple-choice self-reporting inventory that measures the severity of anxiety in adults and adolescents (23). The age range for the measure is from 13 to 80 ; it has been used in peer-reviewed studies with younger adolescents aged 12 and older. The applicability of BAI in Iranian adults has been tested and confirmed (35).

Beck depression inventory (BDI): the Beck depression inventory (BDI, BDI-II), developed by Beck is a 21 -item multiple-choice self-reporting inventory. The BDI is one of the most widely used instruments for measuring the severity of depression. In its current version, the questionnaire is designed for individuals aged 13 and over, and is composed of items relating to symptoms of depression such as hopelessness and irritability, cognitions such as guilt or feelings of being punished, as well as physical symptoms such as fatigue, weight loss and lack of interest in sex (22, 36). The BDI has been used in Iran for those above 15 years and it has been proven to have suitable reliability and validity (37).

\section{Results}

The demographic profile of the study samples in Phase II (Pilot Study) and Phase III are presented in Table 1. In the pilot phase, male students made up $53 \%$ of the respondents while $43 \%$ were female. The age of the respondents ranged from 13 to 19 years, with a mean age of 15.87 years and SD of 1.73. The mean age of males was 15.87 years old with standard deviation (SD) of 1.78 and the mean age of females was 15.87 years old with SD of 1.07. A total of 146 (48.7\%) lived in urban and 154 (51.3\%) lived in rural areas. In the main study the respondents consisted of 308 (46.8\%) males and 271 (53.2\%) females. The respondents were aged between 13 and 19 years, with a mean age of 15.27 years and SD of 1.73 . The mean age of males was 15.48 with SD of 1.07 and the mean age of females was 15.07 with SD of 1.01. In the main study, $54 \%$ lived in urban and $46 \%$ lived in rural areas.

\begin{tabular}{|c|c|c|}
\hline Variables/Data & Pilot Study & Main Study \\
\hline \multicolumn{3}{|l|}{ Gender } \\
\hline Male & $158(52.7)$ & $308(53.2)$ \\
\hline Female & $142(47.3)$ & $271(46.8)$ \\
\hline \multicolumn{3}{|l|}{ Age } \\
\hline 13 & $36(12)$ & $114(19.1)$ \\
\hline 14 & $34(11.3)$ & $98(16.9)$ \\
\hline 15 & $60(20)$ & $112(16.8)$ \\
\hline 16 & $57(16)$ & $115(17.3)$ \\
\hline 17 & $40(13.3)$ & $73(12)$ \\
\hline 18 & $63(21)$ & $32(9.3)$ \\
\hline 19 & $10(3.3)$ & $35(9.8)$ \\
\hline \multicolumn{3}{|l|}{ Location } \\
\hline Urban & $146(48.7)$ & $328(54)$ \\
\hline Rural & $154(51.3)$ & $251(46)$ \\
\hline Total & 300 & 579 \\
\hline
\end{tabular}

${ }^{\mathrm{a}}$ Values are expressed as No. (\%).

\subsection{Result of Pilot Study}

Item Analysis and the development of (A\&D inventory) in pilot study: construct validity was tested using 
EFA by investigating the factor structure and factor loading. Meanwhile, the criterion validity (concurrent validity) was to test how well the A\&D Inventory-p correlates with a previously validated measure, the Beck Anxiety Inventory (BAI) and the Beck Depression Inventory (BDI). The result of the KMO (0.942) and Bartlett's test of sphericity (65776.346) at this stage displayed a satisfactory result. The EFA was conducted using principal component analysis (PCA) with Varimax. Items were retained when the loading was greater than 0.50 . The result of the PCA for A\&D Inventory revealed the presence of two components with eigenvalue exceeding 1.0, which accounted for $52.227 \%$ of total variance explained by $31.328 \%$ for factor 1 and $20.899 \%$ for factor 2 .

The rotated component matrix for A\&D Inventory-p revealed that the first factor was labeled "depression"; a total of 47 items with factor loading ranging from 0.50 from 91 items. This factor accounted for $31.328 \%$ of the scale variance. The second factor was labeled "anxiety"; a total of 24 items with factor loading ranging from 0.50 from 67 items. This factor accounted for $22.405 \%$ (should this be the same with $20.899 \%$ ) of the scale variance. The researcher decided to remove all the items considered as problematic such as those with loadings less than 0.50. A total of 87 items were removed from A\&D Inventory-p and only 71 items were used in the next analysis.

The Cronbach's alpha values obtained for depression and anxiety were 0.978 and 0.967 , respectively. This suggests that the scale can be considered to have high internal consistency. The result of the EFA suggested two variables: anxiety with 24 items and depression with 47 items.

Criterion-related validity in the pilot study: a random sample of 262 adolescent from 300 adolescent in the pilot study were asked to complete the final draft of the A\&D inventory-p and the BDI and BAI questionnaire. After two to four weeks, the samples filled the new instrument again. The relationship between the new instrument (A\&D Inventory-p) with BAI and BDI, respectively, were investigated using Pearson product correlation coefficient. There was a strong positive correlation between the A\&D Inventory-p and BAI scales measuring anxiety $(r=0.72, n$ $=262, \mathrm{P}<0.001)$, and between A\&D Inventory-p and BDI scale measuring depression $(\mathrm{r}=0.696, \mathrm{n}=262, \mathrm{P}<0.001)$. These results showed a high correlation between BAI and anxiety items of A\&D Inventory-p, and BDI and depression items of A\&D Inventory-p. Meanwhile, a low correlation was found between BDI and A\&D Inventory-p $(r=0.310, n$ $=262, \mathrm{P}<0.001)$ measuring anxiety and between BAI and A\&D Inventory-p $(r=0.380, n=262, \mathrm{P}<0.001$ ) measuring depression. Therefore, the high correlation indicates a measure of similar dimension. Meanwhile, the low correlation shows discriminate dimension.

\subsection{Results of the Main Study}

The A\&D Inventory, which comprises of 47 items measuring depression and 24 items measuring anxiety was tested for its validity (construct validity, discriminant validity and criterion validity) using confirmatory factory analysis (CFA). Confirmatory factory analysis was used to confirm and validate the identified factors or dimensions produced by EFA (38). The reliability of the A\&D Inventory was also tested by testing composite reliability, internal consistency and test-retest reliability.

We first tested the normality and presence of outliers. The skewness values were within the range of +2 to -2 and kurtosis value was less than 7, which is an acceptable value for kurtosis, suggesting that data were normally distributed. As for the outliers, we checked for extreme values or outliers using the Mahalanobis distance measure (39). Checking the level of significance (less than .05) in P1 and P2 of Mahalanobis distance measure showed that there were no outliers.

The CFA involves the six following stages. Stage 1: assessing congeneric model validity for "anxiety" of A\&D Inventory. The main purpose at this stage was to assess the unidimensionality of the construct by comparing between models. Model 1 estimates the anxiety inventory using four sub-factors, meanwhile Model 2 estimates the anxiety scale as one congeneric factor. Results of Table 2 show that the values of fit indexes GFI, CFI, NFI and TLI in Model 2 were higher and the RMSEA fit indexes decreased from 0.101 to 0.070 that indicated acceptable fit. Based on the modified EFA Model, Model 2 was considered to be an acceptable Model, which suggest a one-factor model for measuring anxiety.

Stage 2: assessing congeneric model validity for "depression" of A\&D inventory.

Three separate models were tested for the depression scale. Model 1 estimates the depression scale using six subfactors, Model 2 using four sub-factors, and Model 3 estimates depression inventory as one congeneric factor. Results in Table 2 show that Model 3 was found to have the best fit compared to Model 1 and Model 2. The findings suggest a one factor structure for the depression inventory.

Stage 3: construct validity and reliability of the overall anxiety and depression inventory: convergent validity was tested using standardized factor loadings, construct reliability (CR) and average variance extracted (AVE) method. Based on the CFA, items with factor loadings lower than 0.50 were dropped yielding 32 items with 16 items each measuring depression and anxiety. The factor loadings for all the selected items as shown in Table 3 were between 0.55 and 0.82 . According to Hair a factor loading of 0.7 or higher suggests good validity and a loading of 0.6 - 0.7 suggests acceptable validity (40). Construct validity and av- 
Table 2. Nested Model Comparison for Anxiety and Depression in the Anxiety and Depression Inventory

\begin{tabular}{|c|c|c|c|c|c|c|c|c|c|c|}
\hline Variable & & CMIN & DF & $\mathbf{P}$ & CMIN/DF & GFI & CFI & NFI & TUI & RMSEA \\
\hline \multirow{3}{*}{ Anxiety } & Model 1 & 1977.463 & 252 & 0.000 & 7.847 & 0.768 & 0.750 & 0.724 & 0.726 & 0.101 \\
\hline & Model 2 & 868.048 & 104 & 0.000 & 3.347 & 0.945 & 0.956 & 0.949 & 0.933 & 0.070 \\
\hline & $\Delta \chi^{2}$ & 1109.145 & 148 & 0.000 & & & & & & \\
\hline \multirow{4}{*}{ Depression } & Model 1 & 5009.776 & 1034 & 0.000 & 4.845 & 0.722 & 0.794 & 0.754 & 0.784 & 0.076 \\
\hline & Model 2 & 867.358 & 702 & 0.000 & 4.111 & 0.75 & 0.82 & 0.79 & 0.81 & 0.063 \\
\hline & Model 3 & 558.971 & 104 & 0.000 & 3.345 & 0.92 & 0.92 & 0.91 & 0.91 & 0.061 \\
\hline & $\Delta \chi^{2}$ & 4450 & 930 & 0.000 & & & & & & \\
\hline Congeneric Models overall & 2215.322 & 463 & 0.000 & 3.785 & 0.922 & 0.937 & 0.942 & 0.908 & 0.047 & \\
\hline
\end{tabular}

erage variance are shown in Table 3. The CR for the anxiety and depression inventory was 0.962 and 0.973 , respectively. The AVE values for the anxiety and depression inventory were 0.754 and 0.792 , respectively. The AVE values of 0.5 or greater is sufficient evidence for convergent validity (40). The reliability test for internal consistency (Cronbach's alpha) in this stage was 0.901 for depression and 0.897 for anxiety suggesting high internal consistency.

Stage 4: assessing criterion validity for anxiety of A\&D inventory with (BAI) and depression of A\&D inventory (BDI): in this study, the measurement of the congeneric Model across samples was done to test criterion validity of the new instrument (A\&D inventory). The analysis of the different anxiety and depression items used by BDI and BAI was undertaken to test the goodness-of-fit. The correlation between anxiety items of A\&D Inventory with BAI items was 0.80 and the correlation of depression items of A\&D Inventory with BDI items was 0.89 . The results in Table 4 show an adequate fit to the study data as all the fitness indexes were above the acceptable values.

Stage 5: assessing invariance of the CFA measurement model. Measurement of invariance is concerned with whether the test measures something different in one group or in another (41). In this study, the measurement invariance of the model across samples was done to test the factorial validity of the instrument. The analysis of the different groups across either gender was undertaken to test the goodness-of-fit. The model that best fits the data was used to assess each group separately. The CFA results in Table 5 show an adequate fit to the study data, where the goodness-of-fit indexes for both the Female model and Male model were above the fitness indices threshold value. The results also showed no significant difference between males and females in this study. This suggests that the items do not measure differences between male and female respondents.

Stage 6: study reliability (test-retest for reliability for A\&D inventory).

The reliability analysis was run based on a new set of items after deletions for each construct of the instrument. Random samples of 140 students were asked to complete the A\&D inventory (with 32 items) for a second time, two to four weeks after the initial response. The test-retest correlation should be at the $r=0.70$ range $(40)$. Test-retest reliability for 140 participants over two to four weeks reported $\mathrm{r}=\mathbf{0 . 8 8 3}$ for anxiety and $\mathrm{r}=\mathbf{0 . 8 4 1}$ for depression. In general, these results represent a moderate level of reliability.

\section{Discussion}

The A\&D Inventory was developed to measure anxiety and depression among adolescence in Iran. The A\&D Inventory contains 32 items, with 16 items related to anxiety and 16 items related to depression. Five of these items were adapted from other self-report measures and 27 items were new and based on the Iranian culture. The anxiety factor consists of 16 items, 13 items based on the Tripartite Model and three items based on the CCS model. The results provide evidence that the developed instrument achieved sound psychometric properties and is a theoretically valid measure of anxiety and depression among adolescent school students in the Iranian context. The items display strong test-retest reliability and internal consistency and also show composite reliability for both the anxiety and depression inventory. Finally, the A\&D inventory items also show strong convergent validity and good construct validity, discriminant validity, and criterion validity when compared to other self-report measures of depression and anxiety. It is particularly significant that the A\&D Inventory is able to utilize aspects of the Iranian culture to detect symptoms of anxiety and depression like traditional symptom measures, such as the BDI-II and BAI.

These scales also show a strong overlap with traditional measures of depression and should be used for many of the same purposes. For instance, negative affectivity (NA) is common for phenotypic structures of both depression and anxiety. Negative affectivity includes emotions such as sadness, guilt, hostility, uneasiness, fear and selfdissatisfaction $(42,43)$. A symptom of anxiety in this study 
Table 3. Construct Reliability of Anxiety and Depression Inventory

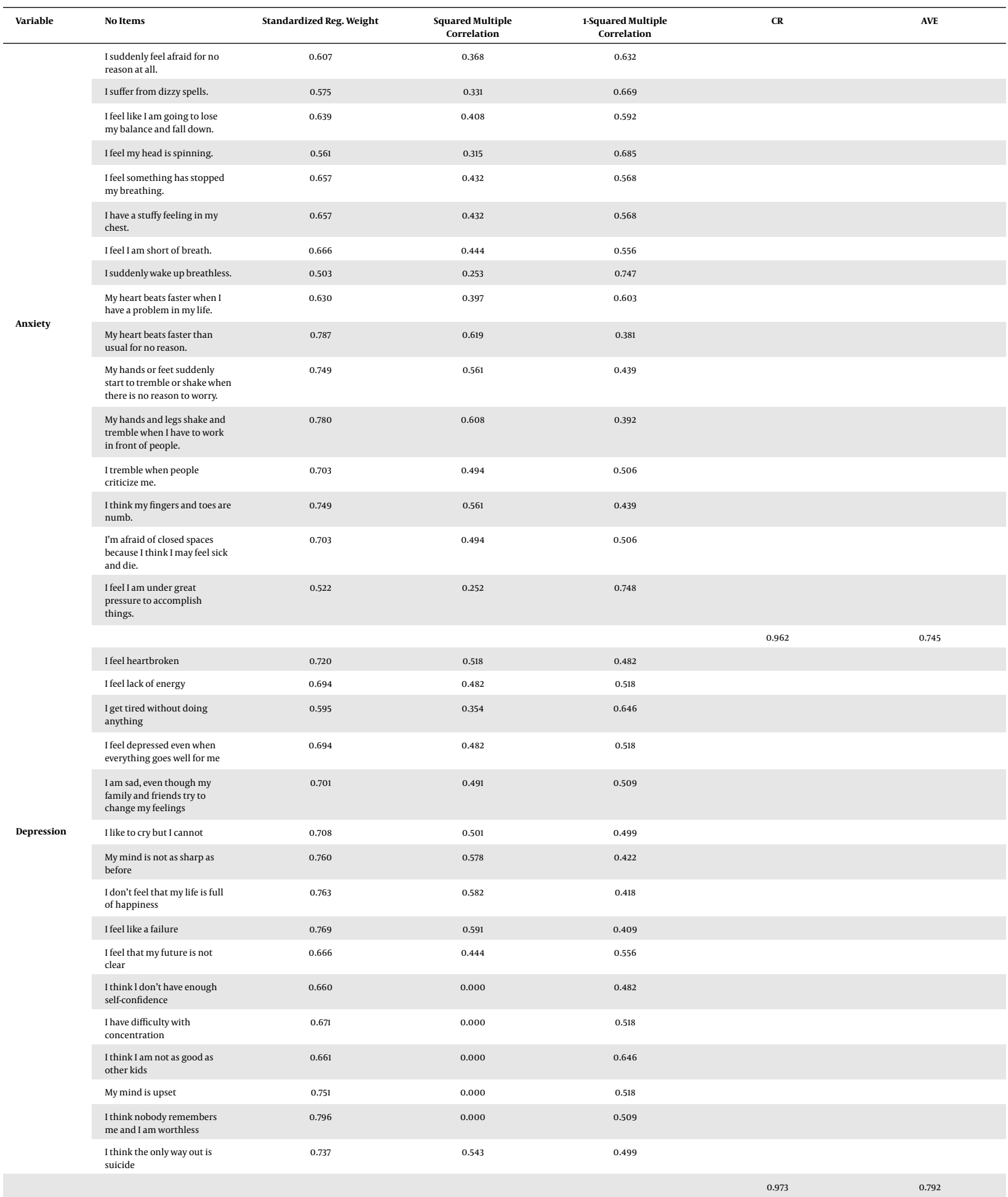


Table 4. Assessing Validity of Congeneric Model in Anxiety and Depression in Anxiety and Depression Inventory with Beck's Anxiety Index and Beck's Depression Index

\begin{tabular}{lcccccccccc}
\hline Variable & CMIN & DF & P & CMIN/DF & GFI & CFI & NFI & TUI & RMR & RMSEA \\
\hline Depression \& BDI & 2176.367 & 629 & 0.000 & 3.180 & 0.916 & 0.894 & 0.917 & 0.90 & 0.545 & 0.077 \\
Anxiety \& BAI & 2160.064 & 628 & 0.000 & 3.195 & 0.908 & 0.917 & 0.905 & 0.90 & 0.498 & 0.075 \\
\hline
\end{tabular}

Table 5. The Construct Model for Anxiety and Depression Inventory for Males and Females

\begin{tabular}{lcccccccc}
\hline Variables & CMIN & DF & P & CMIN/DF & GFI & CFI & NFI & THI \\
\hline Female & 7255.731 & 1701 & 0.000 & 4.261 & 0.927 & 0.960 & 0.926 & 0.945 \\
Male & 7196.731 & 1698 & 0.000 & 4.239 & 0.928 & 0.961 & 0.927 & 0.946 \\
$\Delta \chi^{2}$ & 59 & 3 & 11.34 & & & & \\
\hline
\end{tabular}

too much or fearing something without basis. One item from the A\&D inventory is related to NA (44).

Meanwhile, hyper arousal (PH) includes symptoms such as dizziness, shortness of breath, racing heart and shaky hands. Proponents of the Tripartite Model have hypothesized that this dimension is specific to anxiety disorders. Twelve items from the A\&D inventory consisted of hyper arousal based on the tripartite model. Dizziness includes dizzy spells, feeling of falling down and losing balance, and head spinning (45). Symptoms of shortness of breath in this study meant "stopped breathing, stuffy feeling in chest, shortness of breath, waking up breathless, faster heart beat" and racing heart such as "heart beats faster than usual without reason, hands or feet suddenly start to tremble without reason". When the body deals with a threat, blood pressure and heart rate are increased and sweating is increased (46), while other external signs of anxiety may include trembling and shaky hands (47). Kaplan and Saccuzzo believed that signs of anxiety vary greatly but some common physical symptoms of anxiety include "Shaky hands and legs shake and tremble" and "breathlessness or shortness of breath", which was also found by the current study. In fact, the researcher can say that most of the symptoms of anxiety among Iranian adolescents showed high hyper psychological arousal (48).

As for the cognitive aspect, one of the other symptoms of anxiety in this study was "tremble when people criticize me". Gullotta and Adams found that when adolescents have anxiety, they avoid going out and they panic or feel tense when they are being criticized (49). Critical events would activate these beliefs, which would then create negative automatic thoughts. These cognitive thoughts then lead to symptoms of depression (1). Three items in A\&D inventory belonged to cognitive content-specificity (CCS) theory, and thoughts concerning psychological or psychological threat. Beck found that cognitive content specificity is patent in pure states of anxiety $(23,36,50)$.

The depression factor consists of 16 items, with eight items measuring positive affectivity (PA) based on the tripartite model (positive affact dimension) and eight items based on CCS theory.

Positive Affectivity, which is related to extraversion, is also regarded as a stable and veritable dimension of temperament. Positive Affectivity includes traits such as enthusiasm, excitement seeking, tendency to live in groups and energy. According to the Tripartite Model, people with depression can be distinguished from those with anxiety in that they are identified as having low PA or anhedonia (20). People with anhedonia are characterized as dull, flat, unenthusiastic and disinterested (51). In fact PA has an important role in screening or diagnosing depression. Symptoms of depression are related to sadness such as "heartbroken", lack of energy, such as "tired without doing anything", and anhedonia such as "depressed even when everything goes well or feeling sad even though my family and friends try to change my feelings". Anhedonia is an essential symptom in depression (52). Most of the items in this inventory involved anhedonia symptoms but based on the Iranian culture. Most of the items in the depression questionnaire refer to being sad as: "I am sad" or "I feel sad", but based on Iranian culture, adolescent say: "I feel heartbroken", or "I am sad, even when my family and friends want to help me"

Other depression symptoms such as being upset (eg. "Crying"), sluggish (eg."Not sharp") or not delighted (e.g. "Life is not full of happiness") showed that this disorder usually causes a marked lowering of self-esteem and selfconfidence with increased thoughts of pessimism, hopelessness, and helplessness. In the extreme case, the adolescents may feel excessively and unreasonably guilty (DSMIV-TR). Symptoms of crying are measured by the BDI, but in Iranian culture it is expressed in a different manner. Adolescent in Iran said: "I like to cry but I cannot". Hopelessness is another aspect that was measured in the depression inventory. In fact, in spite of everything being well for Iranian adolescents, they still said that: they felt depressed. 
The researcher believed that this thought is a sign of hopelessness in most of Iranian adolescent, who think that their future is uncertain.

Psychological threats in this inventory, such as "feel like a failure", "future is not clear; "don't have enough selfconfidence", "not as well as other kids", "worthless and suicide" were considered as negative thoughts. Based on DSMIV-TR, "negative thinking" causes depression. This can be extremely dangerous as it can eventually be extremely selfdefeating or lead to suicidal behaviour. The symptom that is most highly correlated with suicidal behaviour is depression. Psychological threat showed symptoms such as difficulty with concentration and mind upset. DSM-IV-TR reported that poor concentration is often an early symptom of this disorder. The depressed person quickly becomes mentally fatigued when asked to read, study, or solve complicated problems. According to Birmaher, depression in adolescents (13 - 18 years) occurs more often in association with other conditions such as learning problems and one of the reasons can be problems in concentration (53). This symptom causes underachievement due to depression (54).

The results of this study have a number of implications in theory and practice. From the theoretical perspective, the generation of items was guided by the operational definition of the construct measured by two theories namely the tripartite model and the cognitive content specificity theory. Clark et al. (21) clarified that the goal of item generation is to create a sample of items that sufficiently cover all relevant content of a construct. Potential items were drawn from multiple sources to ensure the content validity of the new scale. First, the focus was on what was collected and learned from the literature review; this represented the basis of how the instrument would be developed. The information was gathered from various materials, reference books, journals, articles, theses, internet and so forth. This phase was to produce a version of the new instrument. In particular, the A\&D Inventory was developed from two factors of anxiety and depression status, and data on the validity and reliability for its 32 items were provided.

In terms of practical implications, this study was designed to obtain information that would be of value to Iranian adolescents, who come for counseling and assessment of mental health. It can be useful for screening emotional disorders. The A\&D Inventory can give a valid and reliable assessment to better screen and rapidly identify adolescents, who have emotional problems. The A\&D Inventory can also be useful for planning programs in mental health, and could help inform officials about the mental health of adolescent. If further research studies confirmation of the factor structure of the A\&D inventory and assessment of outcome using the A\& D inventory can be uti- lized for developing counseling programs for the Iranian public school system. The A\&D inventory should be easily adaptable for counseling and psychometrics among Iranian adolescent regardless of whether they go to school or not. In addition, the A\&D inventory could be adapted and generalized for use with Iranian college students. It can be modified appropriately for use as part of screening and in tracking the efforts of treatment over time. In health care settings and mental health hospitals where problems with mood and anxiety are common, the A\&D inventory could serve as a battery of instruments that have evidence of validity in measuring anxiety and depression in Iranian context. The A\&D inventory has substantial value for helping to identify problem areas in anxiety and depression of Iranian school students, and can be further tested for use among university students in Iran.

\subsection{Limitation of the Study}

There were some limitations relevant to this study. The limitations are related to the population and instrument. As mentioned, the sample of this study was adolescents from the north of Iran and this limits the generalizability of this study to similar groups in other regions. Although the results of the current study provide promising preliminary reliability and validity on the A\&D Inventory, it is important to note the limitations of the study. The variables showed good inter-item reliability with BAI and BDI, but it should be compared with other well-known scales or inventories in Iran again. This may continue to be a concern in further researches, specifically in a confirmatory factor analysis of the A\&D inventory.

\subsection{Recommendations for Future Research}

In this study, the anxiety and depression inventory (A\&D inventory) was developed, validated, and found to be a reliable and valid tool for measuring emotional disorder constructs among adolescent school students in Iran. Further research replicating this scale (A\&D inventory) should be conducted in the future with larger and different samples to demonstrate the replication of similar results, because any given sample will never perfectly reflect its population. While deviations might be reduced with increases in sample size, they will still occur even with larger samples (43-47). Therefore, it will be important to replicate the factor analysis procedures each time the A\&D Inventory is used in order to demonstrate that the factor structure presented here is not peculiar to this sample and is stable across different samples. Future studies should include the investigation of how the A\&D inventory is related to other anxiety and depression scales or inventories that are used in Iran for assessing mental health scholastic aptitude, and general cognitive development measures where 
Western instruments are still used predominantly. Finally, the normalization of the A\&D inventory can be of help and be useful for Iranian culture in the whole of Iran. Most importantly, although the researcher presented data showing the correlation between the A\&D Inventory and both $\mathrm{BAI}$ and BDI, the researcher has yet examined the inventory in relation to adolescents, who have emotional disorder such as anxiety disorder and/or depression disorder. Hence, further studies are needed to employ the A\&D inventory on Iranian adolescents, who have emotional disorder such as anxiety disorder and/or depression disorder.

\section{Acknowledgments}

This research was part of a PhD thesis at the University of Putra Malaysia. We extend our special thanks to all the teenagers, who participated in this study. We would also like to acknowledge the contribution of the first author's co-workers in Guilan University of Medical Science for helping us with the data collection for the main study.

\section{Footnotes}

Authors' Contribution: Mahnaz Fallahi Khesht-Masjedi conceived and designed the evaluation, collected the data, performed the statistical analysis, interpreted them and drafted the manuscript. Zoharah Omar performed some of the statistical analysis and interpreted them, revised the manuscript and approved the final results. Both authors read and approved the final manuscript.

Financial Disclosure: The authors had no potential conflict of interest including any personal, or other relationships with people or organizations that could inappropriately influence or to be perceived to influence this work.

Funding/Support: The authors had no financial relationships with people or organizations that could in appropriately influence or be perceived to influence this work.

Declaration of Interest: Non declared.

\section{References}

1. Mash E, Wolfe D. Abnormal child psychology. USA: Cengage Learning; 2012.

2. Walker HM, Ramsey E, Gresham FM. Antisocial behavior in school: Evidence-based practices. Wadsworth Publishing Company; 2004.

3. Richmond RL. A guide to psychology and its practice 2009. [8 October 2009]. Available from: http://www.guidetopsychology.com/testing. htm.

4. Emami H, Ghazinour M, Rezaeishiraz H, Richter J. Mental health of adolescents in Tehran, Iran. J Adolesc Health. 2007;41(6):571-6. doi: 10.1016/j.jadohealth.2007.06.005. [PubMed: 18023786].

5. Mundfrom DJ, Shaw DG, Ke TL. Minimum sample size recommendations for conducting factor analyses. Int J Test. 2005;5(2):159-68.
6. Hambleton RK, Merenda PF, Spielberger CD. Adapting educational and psychological tests for cross-cultural assessment. Psychology Press; 2004.

7. Van de Vijver FJR, Leung K. Methodological issues in psychological research on culture. J Cross Cltur Psychol. 2000;31(1):33-51.

8. Hashemian F, Khoshnood K, Desai MM, Falahati F, Kasl S, Southwick S. Anxiety, depression, and posttraumatic stress in Iranian survivors of chemical warfare. JAMA. 2006;296(5):560-6. doi: 10.1001/jama.296.5.560. [PubMed:16882962].

9. Modabber-Nia MJ, Shodjai-Tehrani H, Moosavi SR, Jahanbakhsh-Asli $\mathrm{N}$, Fallahi M. The prevalence of depression among high school and preuniversity adolescents: Rasht, northern Iran. Arch Iran Med. 2007;10(2):141-6.

10. Essau CA, Sakano Y, Ishikawa S, Sasagawa S. Anxiety symptoms in Japanese and in German children. Behav Res Ther. 2004;42(5):601-12. doi: 10.1016/S0005-7967(03)00164-5. [PubMed: 15033504].

11. Modabernia MJ, Shojaie Tehranie H, Falahi M, Faghirpour M. Normalizing SCL-90-R Inventory in Guilan High-School Students. [in Persian]. J Guilan Univ Med Sci. 2010;19(75):58-65.

12. Lari AR, Joghataei MT, Adli YR, Zadeh YA, Alaghehbandan R. Epidemiology of suicide by burns in the province of Isfahan, Iran.J Burn Care Res. 2007;28(2):307-11. doi: 10.1097/BCR.oB013E318031A27F. [PubMed: 17351450].

13. Emami H, Ghazinour M, Rezaeishiraz H, Richter J. Mental health of adolescents in Tehran, Iran. J Adolesc Health. 2007;41:571-6. doi: 10.1016/j.jadohealth.2007.06.005. [PubMed: 18023786].

14. Ishikawa S, Sato H, Sasagawa S. Anxiety disorder symptoms in Japanese children and adolescents. J Anxiety Disord. 2009;23(1):104-11. doi: 10.1016/j.janxdis.2008.04.003. [PubMed: 18555658].

15. Essau CA, Petermann F. Anxiety disorders in children and adolescents: Epidemiology, risk factors and treatment. Routledge; 2013.

16. Jalili SA. Chair of the 8th Annual Congress of Iranian Psychiatric Association 2010. [21 Jun 2010]. Available from: http://www.medlib.ir/ Article/Resource/PDFArticles/65/1738/17839.pdf.

17. Watson D, O'Hara MW, Simms LJ, Kotov R, Chmielewski M, McDadeMontez EA, et al. Development and validation of the Inventory of Depression and Anxiety Symptoms (IDAS). Psychol Assess. 2007;19(3):253-68. doi:10.1037/1040-3590.19.3.253. [PubMed:17845118].

18. Lownsdale $\mathrm{W}$. The development and validity of the psychological symptoms scan. University Northern Illinois University; 2001.

19. Weitkamp K, Romer G, Rosenthal S, Wiegand-Grefe S, Daniels J. German Screen for Child Anxiety Related Emotional Disorders (SCARED): Reliability, Validity, and Cross-Informant Agreement in a Clinical Sample. Child Adolesc Psychiatry Ment Health. 2010;4:19. doi: 10.1186/17532000-4-19. [PubMed: 20591137].

20. Clark LA, Watson D. Tripartite model of anxiety and depression: psychometric evidence and taxonomic implications. J Abnorm Psychol. 1991;100(3):316-36. [PubMed: 1918611].

21. Clark LA, Watson D, Reynolds S. Diagnosis and classification of psychopathology: challenges to the current system and future directions. Annu Rev Psychol. 1995;46:121-53. doi: 10.1146/annurev.ps.46.020195.001005. [PubMed: 7872729].

22. Beck AT, Steer RA, Ball R, Ranieri W. Comparison of Beck Depression Inventories -IA and -II in psychiatric outpatients. J Pers Assess. 1996;67(3):588-97. doi: 10.1207/s15327752jpa6703_13. [PubMed: 8991972].

23. Beck R, Perkins TS. Cognitive content-specificity for anxiety and depression: A meta-analysis. Cogn Ther Res. 2001;25(6):651-63.

24. Netemeyer RG, Bearden WO, Sharma S. Scaling procedures: Issues and applications. Sage Publications; 2003.

25. Reise SP, Waller NG, Comrey AL. Factor analysis and scale revision. Psychol Assess. 2000;12(3):287.

26. Siniscalco MT, Auriat NA. Questionnaire design. Paris: International Institute for Educational Planning; 2005.

27. Carmines EG, Zeller RA. Reliability and validity assessment. Sage publications; 1979 . 
28. Nunnally JC, Bernstein IH, Berge JM. Psychometric theory. 226. JSTOR; 1967.

29. Punch KF. Introduction to social research: Quantitative and qualitative approaches. Sage; 2013.

30. Lancaster GA, Dodd S, Williamson PR. Design and analysis of pilot studies: recommendations for good practice. J Eval Clin Pract. 2004;10(2):307-12. doi: 10.1111/j.2002.384.doc.x. [PubMed: 15189396].

31. Ruxton G, Colegrave N. Experimental design for the life sciences. Oxford University Press; 2010.

32. Festing MFW, Overend P, Das RG, Borja MC, Berdoy M. The Design of Animal Experiments. London: Royal Society of Medicine Press; 2002.

33. Field A. Discovering statistics using SPSS. Sage publications; 2009.

34. Ary D, Jacobs LC, Sorensen CK, Walker D. Introduction to research in education. Cengage Learning; 2013.

35. Kaviani H, Mousavi AS. Psychometric properties of the Persian version of Beck Anxiety Inventory (BAI). [in Persian]. Tehran Univ Med J. 2008;66(2):136-40.

36. Beck R, Benedict B, Winkler A. Depression and anxiety: integrating the tripartite and cognitive content-specificity assessment models. J Psychopathol Behav Assess. 2003;25(4):251-6.

37. Modabernia MJ, Tehrani HS, Fallahi M, Shirazi M, Modabbernia AH Prevalence of depressive disorders in Rasht, Iran: A community based study. Clin Pract Epidemiol Ment Health. 2008;4:20. doi: 10.1186/1745 0179-4-20. [PubMed: 18601715].

38. Byrne BM, Oakland T, Leong FTL, van de Vijver FJR, Hambleton RK, Cheung FM, et al. A critical analysis of cross-cultural research and testing practices: Implications for improved education and training in psychology. Train Educ Profession Psychol. 2009;3(2):94.

39. Byrne BM. Structural equation modeling with AMOS: Basic concepts, applications, and programming. Routledge; 2013.

40. Hair JF. Multivariate data analysis. Prentice Hall; 2009.

41. Kline TJB. Psychological testing: A practical approach to design and evaluation. Sage Publications; 2005.

42. Mineka S, Watson D, Clark LA. Comorbidity of anxiety and unipolar mood disorders. Annu Rev Psychol. 1998;49:377-412. doi: 10.1146/an- nurev.psych.49.1.377. [PubMed: 9496627].

43. Stein MB, Fuetsch M, Muller N, Hofler M, Lieb R, Wittchen HU. Social anxiety disorder and the risk of depression: a prospective community study of adolescents and young adults. Arch Gen Psychiatry. 2001;58(3):251-6. [PubMed: 11231832].

44. Poncelet B. Teen Depression. 2009 [10 December 2009]. Available from: http://teenhealth.about.com/od/emotionalhealth/a/ teendepression.htm.

45. Magee JC, Teachman BA. Distress and recurrence of intrusive thoughts in younger and older adults. Psychol Aging. 2012;27(1):199210. doi: 10.1037/a0024249. [PubMed: 21707184].

46. Smith M. Anxiety attacks and disorders: Guide to the signs, symptoms, and treatment options. ; 2008.

47. Chakraburtty A. Stress, Anxiety, and Depression: How They Affect Your Health."The Cleveland Clinic. ; 2005.

48. Kaplan RM, Saccuzzo DP. Psychological testing: Principles, applications, and issues. Cengage Learning; 2012

49. Gullotta TP, Adams GR. Handbook of Adolescent Behavioral Problems. Springer; 2008.

50. Lamberton A, Oei TP. A test of the cognitive content specificity hypothesis in depression and anxiety. J Behav Ther Exp Psychiatry. 2008;39(1):23-31. doi: 10.1016/j.jbtep.2006.11.001. [PubMed: 17217911].

51. Szabo M. The short version of the Depression Anxiety Stress Scales (DASS-21): factor structure in a young adolescent sample. J Adolesc. 2010;33(1):1-8. doi: 10.1016/j.adolescence.2009.05.014. [PubMed: 19560196].

52. Wetzel JW. Clinical handbook of depression. Gardner Pr; 1984.

53. Birmaher B, Brent D, Aacap Work Group on Quality Issues , Bernet $\mathrm{W}$, Bukstein $\mathrm{O}$, Walter $\mathrm{H}$, et al. Practice parameter for the assessment and treatment of children and adolescents with depressive disorders. J Am Acad Child Adolesc Psychiatry. 2007;46(11):1503-26. doi: 10.1097/chi.ob013e318145ae1c. [PubMed: 18049300].

54. Gilham J. Preventing Teen Depression: A Program Based on Cognitive Behavioral Therapy Gives Adolescents Some Tools To Stave Off Depression.;2011. 